\title{
ACETATOS HOMO E HETEROTRINUCLEARES DE FERRO: UM EXPERIMENTO PARA O LABORATÓRIO DE QUÍMICA DE COORDENAÇÃO ${ }^{*}$
}

\author{
Reginaldo da Silva Santos, Sergio Augusto Venturinelli Jannuzzi e André Luiz B. Formiga* \\ Instituto de Química, Universidade Estadual de Campinas, CP 6154, 13083-970 Campinas - SP, Brasil
}

Recebido em 15/3/10; aceito em 6/5/10; publicado na web em 24/8/10

\begin{abstract}
HOMO AND HETEROTRINUCLEAR IRON ACETATES: AN EXPERIMENT FOR THE COORDINATION CHEMISTRY LABORATORY. Homo and heterotrinuclear acetates are unique compounds having $\mu_{3}$-oxo bridge and many interesting properties of such compounds are derived from this structure. Some undergraduate inorganic textbooks discuss several aspects of these compounds and we present here an undergraduate experiment for the high-yield synthesis of $\left[\mathrm{Fe}_{2} \mathrm{MO}\left(\mathrm{CH}_{3} \mathrm{CO}_{2}\right)_{6}\left(\mathrm{H}_{2} \mathrm{O}\right)_{3}\right]$, with $\mathrm{M}=\mathrm{Fe}^{3+}, \mathrm{Co}^{2+}$ and $\mathrm{Ni}^{2+}$, as well as their characterization using infrared spectroscopy and cyclic voltametry. The proposed experiment gives the opportunity to discuss several concepts of coordination chemistry that follow the characterization techniques, such as: types of acetate coordination, reversibility of electrochemical processes, quelate and trans effects and lability.
\end{abstract}

Keywords: trinuclear iron complexes; coordination chemistry; education.

\section{INTRODUÇÃO}

Os acetatos trinucleares de metais de transição representam uma grande classe de compostos anteriormente denominada de "acetatos básicos" (Figura 1). Atualmente são conhecidos compostos análogos contendo os metais $\mathrm{Mg}, \mathrm{Al}, \mathrm{V}, \mathrm{Cr}, \mathrm{Mn}, \mathrm{Fe}, \mathrm{Co}, \mathrm{Ni}, \mathrm{Cu}, \mathrm{Zn}, \mathrm{Nb}, \mathrm{Mo}$, $\mathrm{Rh}, \mathrm{W}, \mathrm{Ir}$ e Gd. ${ }^{1}$

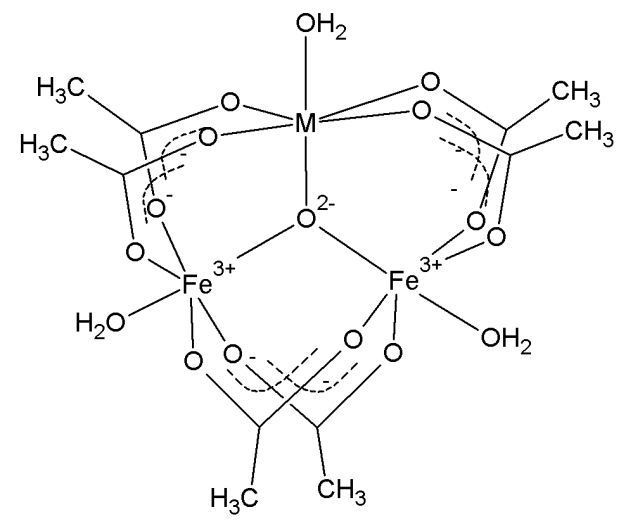

Figura 1. Estrutura dos acetatos básicos trinucleares homonuclear $(M=$ $\left.\mathrm{Fe}^{3+}\right)$ e heteronuclear $\left(\mathrm{M}=\mathrm{Co}^{2+}, \mathrm{Ni}^{2+}\right)$

Esses complexos são conhecidos há décadas e são exemplos de livros texto utilizados amplamente no Brasil como, por exemplo, Advanced Inorganic Chemistry, de Cotton e Wilkinson ${ }^{2}$ e Chemistry of the Elements de Greenwood. ${ }^{3}$ A ênfase dada por Cotton é na ponte $\mu_{3}$-oxo, uma coordenação atípica para a ponte e contestada no início do século passado, mas que foi confirmada com a resolução da estrutura cristalina por difratometria de raios X para o trinuclear de crômio, a primeira a ser obtida para a série dos acetatos básicos pelo famoso químico Leslie Orgel. ${ }^{4} \mathrm{O}$ próprio Cotton foi o primeiro a resolver a estrutura cristalina de um acetato de rutênio, em 1972. ${ }^{5}$ No livro de Greenwood, os trinucleares de Mo e W são citados para

*e-mail: formiga@iqm.unicamp.br

${ }^{\#}$ Em homenagem ao Prof. Dr. Henrique E. Toma por ocasião do seu $60^{\circ}$ aniversário. exemplificar a existência de ligações metal-metal e para evidenciar as diferenças entre estes e o trinuclear de crômio, onde as interações entre os metais são consideradas fracas.

Dentre essa classe de compostos, o cluster de rutênio é, sem dúvida, aquele melhor estudado do ponto de vista eletroquímico, pois apresenta o comportamento redox mais versátil sendo possível acessar até cinco estados de valência mista (uma revisão sobre esses trinucleares de rutênio pode ser encontrada em Química Nova). ${ }^{6}$ Essas propriedades têm motivado sua aplicação em dispositivos supramoleculares para diversos fins, como catálise, ${ }^{7}$ eletrocatálise ${ }^{8}$ e conversão de energia. ${ }^{9}$

Devido ao alto valor do reagente de rutênio (tipicamente $\mathrm{RuCl}_{3}$. $\mathrm{nH}_{2} \mathrm{O}$ ), a síntese desses complexos em larga escala num laboratório didático fica inviabilizada, apesar disso, outros metais como $\mathrm{Fe}, \mathrm{Ni}$, Co são também adequados à síntese. Julgamos o estudo dessa classe de compostos importante para uma formação mais completa dos discentes, uma vez que através dele podemos abordar temas correntes na química de coordenação, como estabilidade termodinâmica, intervalência, labilidade, efeito e influência trans, magnetismo, etc. Além das questões voltadas aos aspectos fundamentais da química de coordenação, esses compostos têm encontrado aplicações recentes e esse aspecto também pode ser explorado em sala de aula.

Dentre as diversas estruturas conhecidas para os metais da primeira série de transição, duas se destacam em função de suas recentes aplicações em materiais de alto valor agregado. A primeira delas é composta por trinucleares de crômio que deram origem a uma rede metalorgânica (Metal-Organic Framework, ou simplesmente MOF) com íons tereftalato conhecida como MIL-101. Esse material apresenta elevada atividade catalítica em reações de sililação e também como suporte para paládio que, em reações de hidrogenação, se mostrou mais estável e mais ativo do que paládio suportado em carbono ativado. ${ }^{10}$ Este material também se mostrou bastante eficiente como um adsorvedor para gases como $\mathrm{CH}_{4}, \mathrm{CO}_{2}$ e $\mathrm{H}_{2} \mathrm{~S}$, ampliando o interesse nesse tipo de estrutura. ${ }^{11}$

$\mathrm{O}$ trinuclear de manganês tem sido usado como um precursor para a síntese de magnetos moleculares (Single-Molecule Magnets, ou simplesmente $S M M$ ) com nuclearidade maior variando de $\mathrm{Mn}_{12}$ a $\mathrm{Mn}_{19}$ que estão entre os mais promissores para serem utilizados em dispositivos magnéticos. ${ }^{12}$

Os clusters de acetato de ferro foram isolados pela primeira vez em 1909 por Weinland e Gussmann, ${ }^{13}$ embora naquele tempo 
eles não estivessem aptos a propor a fórmula correta. Os primeiros exemplos de clusters trinucleares de metais mistos foram sintetizados por Weinland e Holtmeier, ${ }^{14}$ mas não foram reconhecidos naquela época. Mais tarde, estudos de Blake e colaboradores mostraram por investigações de difração de raios $\mathrm{X}$ que os produtos obtidos pelo procedimento de Weinland foram de fato espécies trinucleares $\left[\mathrm{Fe}_{2}^{\mathrm{III}} \mathrm{M}^{\mathrm{II}} \mathrm{O}\left(\mathrm{CH}_{3} \mathrm{CO}_{2}\right)_{6}\left(\mathrm{H}_{2} \mathrm{O}\right)_{3}\right]$, sendo que $\mathrm{M}=\mathrm{Co}$ ou Ni. ${ }^{15}$

Recentemente, a atividade catalítica de trinuclear de ferro de valência mista frente à oxidação de ácido ascórbico e um derivado lipídico (6-palmitato) foi reportada. O composto mimetiza a estratégia empregada pela natureza na oxidação de lipídeos sem uso de enzima, apenas com ácido ascórbico e um sal de ferro. ${ }^{16} \mathrm{~A}$ vantagem de tais complexos em relação ao oxigênio molecular é a baixa barreira energética para a conversão do estado tripleto para singleto. As propriedades magnéticas dos trinucleares de ferro, por sua vez, foram vastamente estudadas por Tuchagues e colaboradores. ${ }^{17}$ Apesar de o $\mathrm{Fe}^{3+}$ em campo fraco ter momento magnético total de spin igual a 5/2, o trinuclear $\left(\mathrm{Fe}_{3} \mathrm{O}^{7+}\right)$ tem $\mathrm{S}=1 / 2$, indicando que efetivamente os núcleos metálicos interagem entre si através das pontes $\mu_{3}$-oxo e $\mu_{2}$-acetato.

O método clássico de obtenção dos trinucleares mistos de $\mathrm{Fe}_{2}^{\mathrm{III}} \mathrm{M}^{\mathrm{II}}$, sendo $\mathrm{M}$ um metal de transição, consiste em uma reação de uma mistura de um sal de ferro(III) e um sal metálico divalente com acetato de sódio em solução aquosa ou alcoólica. ${ }^{15}$ Essa metodologia normalmente leva à mistura de produtos homo e heteronucleares, $\mathrm{o}$ que diminui o rendimento da síntese. Recentemente, Nunes e colaboradores ${ }^{13}$ descreveram uma simplificação dessa rota de síntese e que também apresenta rendimentos próximos de $90 \%$ em média, o que motivou a utilização dessas sínteses em nosso laboratório didático.

\section{Conteúdos que podem ser explorados}

O primeiro aspecto que pode ser explorado com os alunos é a estrutura dessa classe de compostos. Os complexos apresentam os íons metálicos hexacoordenados, com dois tipos de pontes e ligantes terminais. Os íons acetatos formam pontes entre dois íons metálicos, além de um átomo de oxigênio central, em ponte entre os três íons, tal como mostrado na Figura 1.

Cabe ressaltar que, usualmente, o oxigênio que faz três ligações apresenta hibridização $\mathrm{sp}^{3}$, como é o caso do íon hidroxônio $\left(\mathrm{H}_{3} \mathrm{O}^{+}\right)$, cuja estrutura se assemelha à amônia. No entanto, o oxigênio localizado sobre o eixo de simetria $\mathrm{C}_{3}$ desses compostos é planar, ${ }^{18}$ permitindo que seu orbital $\mathrm{p}_{\mathrm{z}}$, perpendicular ao plano das ligações com o metal, tenha simetria adequada para se combinar com orbitais $3 d$ dos íons, num mecanismo semelhante ao de retrodoação. Uma vez que os três núcleos metálicos têm orbitais $d$ com simetria adequada, forma-se um orbital ligando os três metais através da ponte oxo. Esse tipo de coordenação para a ponte oxo é exclusivamente encontrado nessa classe de compostos. ${ }^{19,20}$

O modo de coordenação dos acetatos em ponte é facilmente verificado usando espectroscopia no infravermelho, uma vez que os modos de estiramento simétrico e assimétrico dos acetatos coordenados são bastante diferentes do acetato livre. Além disso, os modos da unidade $\mathrm{M}_{3} \mathrm{O}$ aparecem em região de $500-700 \mathrm{~cm}^{-1}$ e podem ser observados em espectrômetros convencionais usando pastilhas de $\mathrm{KBr}$, sem maiores problemas.

A ponte $\mu_{3}$-oxo é responsável pelas propriedades atípicas desses compostos quando comparadas aos complexos mononucleares dos íons no mesmo estado de oxidação. A primeira delas são os estados de intervalência que podem ser obtidos. Os alunos devem ser estimulados a calcular os estados de oxidação dos compostos sintetizados que, na estrutura $\left[\mathrm{M}_{2} \mathrm{M}^{\prime} \mathrm{O}\left(\mathrm{CH}_{3} \mathrm{CO}_{2}\right)_{6}\left(\mathrm{H}_{2} \mathrm{O}\right)_{3}\right]$ equivalem a $8 / 3$ ou $(3+3+2) / 3$, isto é, dois núcleos metálicos estão em estado de oxidação III e um em II. Devido à deslocalização eletrônica formada pela ponte oxo, não é possível precisar qual núcleo apresenta qual estado de oxidação num cluster homometálico, principalmente em compostos da segunda série de transição.

Outro aspecto que pode ser explorado são reações de substituição dos ligantes terminais em que as reações de troca dos ligantes nesses complexos trinucleares se tornam $10^{2}-10^{4}$ vezes mais rápidas quando comparadas aos complexos mononucleares. ${ }^{21}$ Esse aumento na velocidade das reações de troca dos ligantes está associado à presença da ponte $\mu_{3}$-oxo, que provoca um efeito trans bastante acentuado via um mecanismo dissociativo (D) ou troca dissociativa $\left(\mathrm{I}_{\mathrm{d}}\right)$, também observado em outros complexos que possuem pontes oxo angulares. ${ }^{22}$

Associadas às reações de substituição dos ligantes terminais também ocorrem as substituições dos metais nessas estruturas trinucleares com metais da primeira série de transição. Essa estratégia foi muito bem explorada por Nunes e colaboradores ${ }^{13}$ na síntese de compostos heteronucleares, que motivou este trabalho. Nestas reações usando como reagente os trinucleares homonucleares com os três metais no estado de oxidação III, apenas um dos metais é trocado por outro metal no estado de oxidação II. A mudança na carga total do complexo (o produto é neutro) provoca a precipitação em meio aquoso, deslocando o equilíbrio para a obtenção do produto.

Do ponto de vista da labilidade dos metais nestes compostos, o procedimento experimental proposto é um bom exemplo das diferenças entre os íons em estados de oxidação II e III e entre metais da $1^{\mathrm{a}}$ e da $2^{\mathrm{a}}$ séries de transição. Um experimento de voltametria cíclica em meio aquoso permite determinar a redução do trinuclear, uma reação irreversível em virtude da maior labilidade dos íons $\mathrm{Fe}^{2+} \mathrm{em}$ relação aos íons $\mathrm{Fe}^{3+}$. Com o auxílio de referências complementares aqui citadas, os alunos podem confirmar essa grande diferença entre as labilidades, incluindo comparações com outros trinuclares de metais da segunda série de transição.

\section{PARTE EXPERIMENTAL}

Todos os procedimentos de síntese foram planejados para serem realizados num único dia de trabalho, de modo que três compostos trinucleares possam ser obtidos na forma sólida num período de $8 \mathrm{~h}$ de laboratório. Facilmente, o professor pode adaptar o procedimento para duas aulas de $4 \mathrm{~h}$, sendo a primeira para a obtenção do trinuclear homometálico e a segunda para a obtenção dos compostos heterometálicos. No Instituto de Química da Unicamp os alunos utilizaram mais uma aula de $8 \mathrm{~h}$ para a realização dos procedimentos de análise por infravermelho e voltametria cíclica.

\section{Obtenção do $\left[\mathrm{Fe}_{3} \mathrm{O}\left(\mathrm{CH}_{3} \mathrm{CO}_{2}\right)_{6}\left(\mathrm{H}_{2} \mathrm{O}\right)_{3}\right] \mathrm{CH}_{3} \mathrm{CO}_{2}$}

Em um béquer de $50 \mathrm{~mL}$ foram dissolvidos $1,5 \mathrm{~g}$ de $\mathrm{FeCl}_{3} \cdot 6 \mathrm{H}_{2} \mathrm{O}$ em aproximadamente $5 \mathrm{~mL}$ de água desionizada. Em seguida, foi acrescentado excesso de $\mathrm{NH}_{4} \mathrm{OH}$ concentrado com leve agitação. $\mathrm{O}$ $\mathrm{Fe}(\mathrm{OH})_{3}$ sólido formado foi filtrado em um funil de Büchner e lavado 6 vezes com aproximadamente $50 \mathrm{~mL}$ de água desionizada, até não ser mais observada a presença de cloreto no filtrado (teste negativo com solução de nitrato de prata).

Em um béquer de $50 \mathrm{~mL}$, sob agitação e aquecimento brando $\left(\sim 60{ }^{\circ} \mathrm{C}\right)$, o sólido obtido foi dissolvido em $15 \mathrm{~mL}$ de uma solução aquosa $50 \%$ de ácido acético glacial. Nessa etapa é formada uma solução castanho-alaranjada. Mantendo o mesmo aquecimento, a solução foi deixada evaporar até a obtenção de um pó alaranjado de $\left[\mathrm{Fe}_{3} \mathrm{O}\left(\mathrm{CH}_{3} \mathrm{CO}_{2}\right)_{6}\left(\mathrm{H}_{2} \mathrm{O}\right)_{3}\right] \mathrm{CH}_{3} \mathrm{CO}_{2}$. Nessa etapa, foram tomadas as devidas precauções para evitar a decomposição térmica da amostra, mantendo-se controle rigoroso da temperatura e também se evitando prolongar o aquecimento após a completa secagem da amostra.

O material obtido foi pesado e dividido em 3 partes iguais, sendo 
uma reservada e deixada em dessecador contendo sílica gel pelo período de uma semana sob vácuo. A partir dessa amostra foi possível estimar o percentual de umidade/solvente ainda presente no material.

A partir de 2/3 da massa do sólido laranja obtido na primeira etapa da síntese, foram preparados os trinucleares heteronucleares com cobalto $\left[\mathrm{Fe}_{2} \mathrm{CoO}\left(\mathrm{CH}_{3} \mathrm{CO}_{2}\right)_{6}\left(\mathrm{H}_{2} \mathrm{O}\right)_{3}\right]$ e níquel $\left[\mathrm{Fe}_{2} \mathrm{NiO}\left(\mathrm{CH}_{3} \mathrm{CO}_{2}\right)_{6}\left(\mathrm{H}_{2} \mathrm{O}\right)_{3}\right]$.

\section{Obtenção do $\left[\mathrm{Fe}_{2} \mathrm{CoO}\left(\mathrm{CH}_{3} \mathrm{CO}_{2}\right)_{6}\left(\mathrm{H}_{2} \mathrm{O}\right)_{3}\right]$}

Em um béquer de $25 \mathrm{~mL} \mathrm{1/3}$ da massa do $\left[\mathrm{Fe}_{3} \mathrm{O}\left(\mathrm{CH}_{3} \mathrm{CO}_{2}\right)_{6}\left(\mathrm{H}_{2} \mathrm{O}\right)_{3}\right]$ $\mathrm{CH}_{3} \mathrm{CO}_{2}$ foi dissolvido em aproximadamente $3 \mathrm{~mL}$ de $\mathrm{H}_{2} \mathrm{O}$ desionizada. Em outro béquer de $25 \mathrm{~mL}$ foi dissolvida igual massa de $\mathrm{Co}\left(\mathrm{CH}_{3} \mathrm{CO}_{2}\right)_{2} \cdot 4 \mathrm{H}_{2} \mathrm{O}$ em aproximadamente $3 \mathrm{~mL}$ de $\mathrm{H}_{2} \mathrm{O}$ desionizada. Após a dissolução completa de ambos sólidos, duas soluções são misturadas e deixadas sob suave agitação e aquecimento $\left(\sim 50{ }^{\circ} \mathrm{C}\right)$ por 10 min. Em seguida, a mistura foi resfriada e o sólido separado por filtração em funil de Büchner. O sólido obtido foi mantido em dessecador contendo sílica por uma semana.

\section{Obtenção dos complexos $\left[\mathrm{Fe}_{2} \mathrm{MO}\left(\mathrm{CH}_{3} \mathrm{CO}_{2}\right)_{6}\left(\mathrm{H}_{2} \mathrm{O}\right)_{3}\right], \mathrm{M}=\mathrm{Ni}, \mathrm{Zn}$}

Na síntese dos outros complexos heterometálicos, foi realizado um procedimento semelhante ao apresentado na sessão acima, substituindo-se o sal de $\mathrm{Co}^{2+}$ por $\mathrm{Ni}\left(\mathrm{CH}_{3} \mathrm{CO}_{2}\right)_{2} \cdot 4 \mathrm{H}_{2} \mathrm{O}$ e $\mathrm{Zn}\left(\mathrm{CH}_{3} \mathrm{CO}_{2}\right)_{2} \cdot 2 \mathrm{H}_{2} \mathrm{O}$. Os sólidos obtidos foram mantidos em dessecador contendo sílica por uma semana. Após esse período, eles foram pesados e o percentual de água foi estimado.

\section{Caracterização das amostras}

Todos os sólidos sintetizados foram caracterizados por espectroscopia na região do infravermelho com transformada de Fourier (FTIR) e voltametria cíclica.

As análises de FTIR foram realizadas na região entre 2200 e 400 $\mathrm{cm}^{-1}$. Essa análise foi realizada utilizando aproximadamente $1 \mathrm{mg}$ da amostra suportada em partilhas de $\mathrm{KBr}$. O experimento de voltametria cíclica foi realizado empregando um potenciostato da Microquímica modelo MQPG-01 empregando um sistema de três eletrodos. Como eletrodos de trabalho e contraeletrodo foram usados um disco e fio de platina, respectivamente. $\mathrm{Um}$ eletrodo $\mathrm{Ag} / \mathrm{AgCl}$ foi usado como eletrodo de referência. As curvas de voltametria cíclica foram obtidas nas velocidades de 50, 75 e $100 \mathrm{mV} / \mathrm{s}$ para soluções dos trinucleares na concentração de $3,0 \times 10^{-3} \mathrm{~mol} \mathrm{~L}^{-1}$ tendo $\mathrm{KCl}\left(0,1 \mathrm{~mol} \mathrm{~L}^{-1}\right)$ como eletrólito suporte. As curvas foram obtidas em uma única varredura no sentido catódico numa faixa de potencial entre 0,90 e $-0,45 \mathrm{~V}$.

\section{RESULTADOS E DISCUSSÃO}

\section{Sínteses}

Todos os procedimentos sintéticos são acessíveis a um aluno que tenha realizado uma disciplina básica de técnicas de laboratório. No experimento realizado, sintetizou-se primeiramente o composto homometálico de $\mathrm{Fe}^{\mathrm{III}}\left[\mathrm{Fe}_{3} \mathrm{O}\left(\mathrm{CH}_{3} \mathrm{CO}_{2}\right)_{6}\left(\mathrm{H}_{2} \mathrm{O}\right)_{3}\right] \mathrm{CH}_{3} \mathrm{CO}_{2}$ (ou simplesmente $\mathrm{Fe}_{3} \mathrm{O}^{+}$). Essa síntese é descrita pelas reações de formação do hidróxido de ferro(III) e pela reação com ácido acético glacial. O professor pode questionar os alunos sobre a importância de se obter primeiramente o hidróxido e, também, sobre a necessidade de se eliminar cloreto.

Os rendimentos obtidos pelos alunos para a reação foram baseados na massa de $\mathrm{FeCl}_{3} \cdot 6 \mathrm{H}_{2} \mathrm{O}$ utilizada, obtendo-se em média $70 \%$.

A partir do $\mathrm{Fe}_{3} \mathrm{O}^{+}$realizaram-se reações de substituição de um núcleo metálico. A primeira evidência da substituição do $\mathrm{Fe}^{3+}$ pelo
$\mathrm{Co}^{2+}$ foi a mudança da cor, pois o sólido de partida era laranja e passou a marrom-avermelhado com a incorporação do $\mathrm{Co}^{2+}$.

Outra mudança importante observada com a incorporação do $\mathrm{Co}^{2+}$ foi na solubilidade do composto. Ao substituir um íon metálico com carga $3+$ por um íon $2+$, a carga do composto anula-se, justificando que o $\mathrm{Fe}_{3} \mathrm{O}^{+}$seja solúvel em água e o $\left[\mathrm{Fe}_{2} \mathrm{CoO}\left(\mathrm{CH}_{3} \mathrm{CO}_{2}\right)_{6}\left(\mathrm{H}_{2} \mathrm{O}\right)_{3}\right]$ $\left(\mathrm{Fe}_{2} \mathrm{CoO}\right)$ seja pouco solúvel.

$\mathrm{O}$ rendimento foi baseado na massa de $\mathrm{Fe}_{3} \mathrm{O}^{+}$utilizada, que em média correspondeu a mais de $95 \%$.

A reação do $\mathrm{Fe}_{3} \mathrm{O}^{+}$com o acetato de zinco rendeu o $\left[\mathrm{Fe}_{2} \mathrm{ZnO}\left(\mathrm{CH}_{3} \mathrm{CO}_{2}\right)_{6}\left(\mathrm{H}_{2} \mathrm{O}\right)_{3}\right]$, cuja coloração foi laranja, muito semelhante com o composto de partida. Novamente o rendimento foi calculado sobre a massa do precursor homometálico de ferro(III), que em média correspondeu a mais de $95 \%$.

Nessas etapas de síntese, o aluno foi estimulado a propor uma fórmula geral para os complexos obtidos e, de modo geral, os alunos propuseram a água ou o acetato como ligantes terminais. $\mathrm{O}$ aluno foi orientado a utilizar os espectros de infravermelho como uma ferramenta para: 1) verificar a presença dos íons acetato; 2) propor o modo de coordenação, a saber, livre (ânion), monodentado (caso seja ligante terminal) e bidentado (como ponte).

\section{Análises}

A Figura 2 apresenta as curvas FTIR obtidas para as amostras de trinucleares heteronucleares de $\mathrm{Ni}$, Co e $\mathrm{Zn}$ comparadas ao trinuclear homonuclear de Fe. Dependendo do conhecimento dos alunos em teoria de grupo, o professor poderá promover uma discussão a respeito dos modos normais de vibração do grupo pontual $\mathrm{D}_{3 \mathrm{~h}}$ e suas atividades no infravermelho. São esperados nove modos vibracionais normais $\mathrm{A}_{1}$ (ativo no Raman), cinco A" (inativo), seis A' (inativo), nove A", (ativo no infravermelho), dezesseis E' (Raman e infravermelho) $\mathrm{e}^{2}$ treze E" (Raman). ${ }^{23}$

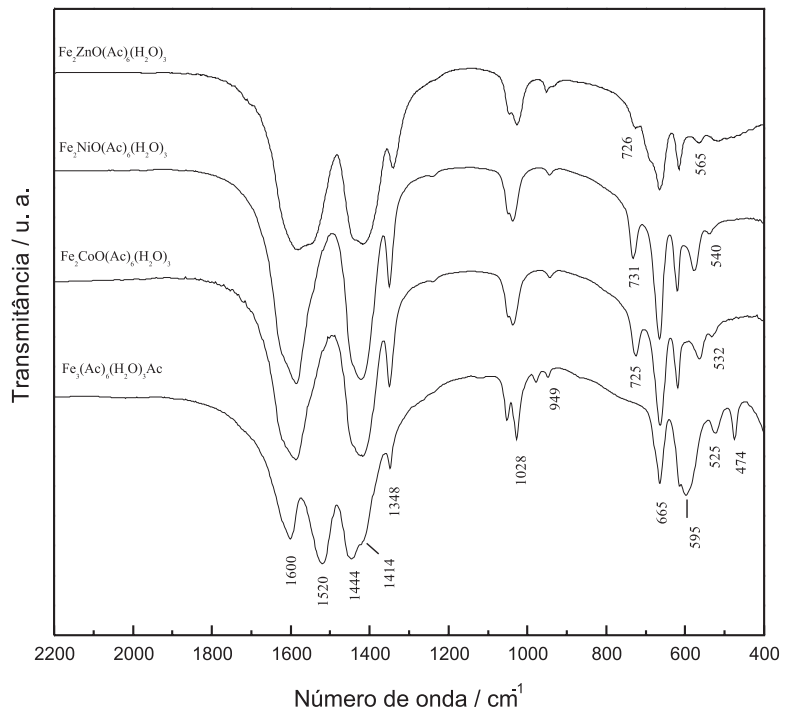

Figura 2. Espectros FTIR obtidos para compostos trinucleares de Fe e dos heteronucleares de $\mathrm{Ni}$, Co e Zn em pastilha de $\mathrm{KBr}$, obtidos por alunos durante a aula experimental

O professor poderá dividir a discussão da espectroscopia infravermelho em duas partes: vibrações dos grupos acetato (ligado e livre) e da unidade $\mathrm{M}_{3} \mathrm{O}$. As duas bandas mais intensas em 1600 e 1444 $\mathrm{cm}^{-1}$ do espectro do $\mathrm{Fe}_{3} \mathrm{O}$ correspondem aos estiramentos assimétrico e simétrico dos carboxilatos ligados em ponte, respectivamente. As bandas desses modos deslocam-se para 1587 e $1420 \mathrm{~cm}^{-1}$ quando um 
Tabela 1. Número de onda e atribuição das bandas no infravermelho dos complexos trinuleares de acetato. Atribuições retiradas das refs. 23 e 24

\begin{tabular}{lccccccccc}
\hline Composto & $v_{\text {ass }}(\mathrm{COO})$ & $v_{\text {sim }}(\mathrm{COO})$ & $\delta\left(\mathrm{CH}_{3}\right)$ & $\rho_{\mathrm{r}}\left(\mathrm{CH}_{3}\right)$ & $v(\mathrm{CC})$ & $\delta(\mathrm{OCO})$ & $\pi(\mathrm{COO})$ & $\rho_{\mathrm{r}}(\mathrm{COO})$ & $v_{\text {ass }}\left(\mathrm{M}_{3} \mathrm{O}\right)$ \\
\hline $\mathrm{Fe}_{3} \mathrm{O}$ & 1600 & 1444 & 1348 & $1053^{\mathrm{a}} 1028^{\mathrm{b}}$ & 949 & 665 & 615 & 525 & 595 \\
$\mathrm{Fe}_{2} \mathrm{Co}$ & 1587 & 1417 & 1348 & $1051^{\mathrm{a}} 1036^{\mathrm{b}}$ & 943 & 665 & 619 & 532 & 725 \\
$\mathrm{Fe}_{2} \mathrm{Ni}$ & 1587 & 1421 & 1350 & $1051^{\mathrm{a}} 1038^{\mathrm{b}}$ & 943 & 665 & 619 & 540 & 731 \\
$\mathrm{Fe}_{2} \mathrm{Zn}$ & 1581 & 1418 & 1341 & $1045^{\mathrm{a}} 1027^{\mathrm{b}}$ & 951 & 666 & 615 & 578 & 726 \\
$\mathrm{CH}_{3} \mathrm{CO}_{2}^{-}$ & 1520 & 1414 & 1348 & & 978 & & 615 & 474 & \\
\hline
\end{tabular}

${ }^{\mathrm{a}}$ simetria $\mathrm{B}_{2} \cdot{ }^{\mathrm{b}}$ simetria $\mathrm{B}_{1}$.

metal é trocado. $\mathrm{O}$ espectro do $\mathrm{Fe}_{3} \mathrm{O}$ apresenta ainda as bandas desses modos vibracionais do acetato livre (contraíon) em 1520 e $1414 \mathrm{~cm}^{-1}$. Outra distinção entre acetatos ligado e livre é notada na região em torno de $950 \mathrm{~cm}^{-1}$, onde a banda de baixa intensidade é relativa ao estiramento C-C. $\mathrm{O}$ espectro do $\mathrm{Fe}_{3} \mathrm{O}$ apresenta duas bandas nessa região, enquanto que os dos heterometálicos, apenas uma, por terem somente acetatos ligados. Outras bandas e as respectivas atribuições são apresentadas na Tabela 1.

As vibrações da unidade $\mathrm{M}_{3} \mathrm{O}$ ativas no infravermelho são os estiramentos assimétricos (modo duplamente degenerado E', ativo também no Raman) e a deformação angular simétrica $\left(\mathrm{A}_{2}{ }_{2}\right)$. $\mathrm{O}$ modo de estiramento totalmente simétrico $\mathrm{A}_{1}{ }_{1}$ é ativo apenas no Raman. Uma vez que os espectros foram registrados a partir de 400 $\mathrm{cm}^{-1}$, somente a banda do estiramento assimétrico foi investigada. $\mathrm{O}$ trinuclear de ferro apresenta banda $v_{\text {ass }}\left(\mathrm{Fe}_{3} \mathrm{O}\right)$ em $595 \mathrm{~cm}^{-1}$. Quando há substituição de um $\mathrm{Fe}^{\mathrm{III}}$ por $\mathrm{M}^{\mathrm{II}}$, $(\mathrm{M}=\mathrm{Co}, \mathrm{Ni}, \mathrm{Zn})$ ocorre abaixamento da simetria da molécula, de $\mathrm{D}_{3 \mathrm{~h}}$ para $\mathrm{C}_{2 \mathrm{v}}$, isso acarreta perda da degenerescência dos modos E' e o surgimento de duas bandas referentes ao $v_{\text {ass }}\left(F_{2} \mathrm{MO}\right)$ em cerca de 725 e $570 \mathrm{~cm}^{-1}$ no espectro dos compostos heterometálicos. Essa segunda banda em geral é fraca e fica encoberta, segundo a literatura. ${ }^{24}$

\section{Voltametrias cíclicas}

Os voltamogramas cíclicos obtidos para os trinucleares homo e heteronucleares, assim como para os precursores usados na síntese, estão apresentados na Figura 3. Os voltamogramas obtidos para os precursores são totalmente distintos daqueles apresentados por todos os trinucleares. Os voltamogramas apresentam picos catódicos em -140, -160 e -190 mV para os trinucleares de Fe, Co e Ni, respectivamente. $\mathrm{O}$ trinuclear de $\mathrm{Zn}$ além de não apresentar um pico definido, possui baixa corrente anódica, quando comparado aos outros trinucleares. As diferenças significativas entre os voltamogramas dos reagentes e dos produtos obtidos devem ser exploradas em sala de aula como mais uma evidência para os alunos confirmarem o sucesso da síntese. De fato, os grupos de alunos que falharam no procedimento sintético obtiveram voltamogramas semelhantes aos reagentes.

Diversos aspectos podem ser explorados com os alunos na interpretação dos voltamogramas cíclicos dos trinucleares. A ausência de pico anódico em todos os casos revela que ocorre uma reação química acoplada que sucede o processo de redução. Segundo Vrubel e colaboradores, ${ }^{13}$ a redução do composto homonuclear ocorre por um mecanismo bieletrônico e provoca a fragmentação da estrutura do trinuclear. A observação dos produtos de decomposição no sentido anódico foi raramente observada pelos alunos, porém, de modo geral, eles puderam concluir sobre a ocorrência de reações acopladas que consumiam o produto da redução, indisponibilizando-o para o processo reverso. Qualitativamente os alunos foram levados a observar as diferenças entre os voltamogramas do trinuclear homonuclear $\left(\mathrm{Fe}_{3} \mathrm{O}^{+}\right)$ e os trinucleares heteronucleares de cobalto e níquel. Observa-se que a área da onda de redução para o composto homonuclear é maior que nos outros casos, o que pode ser compreendido como um processo bieletrônico e, nos outros casos, monoeletrônico, uma vez que um

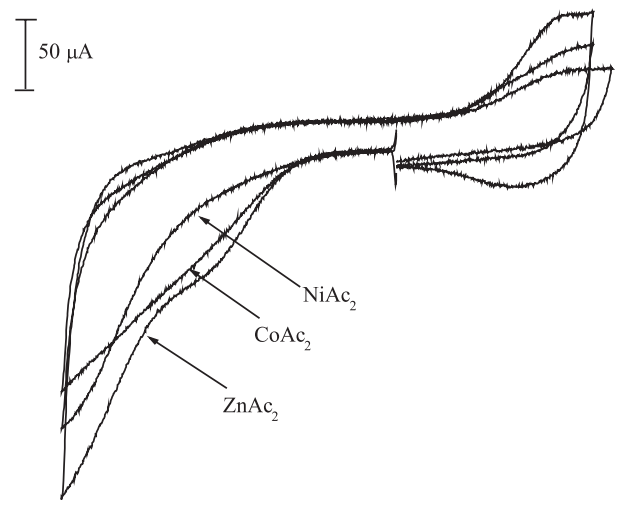

a)
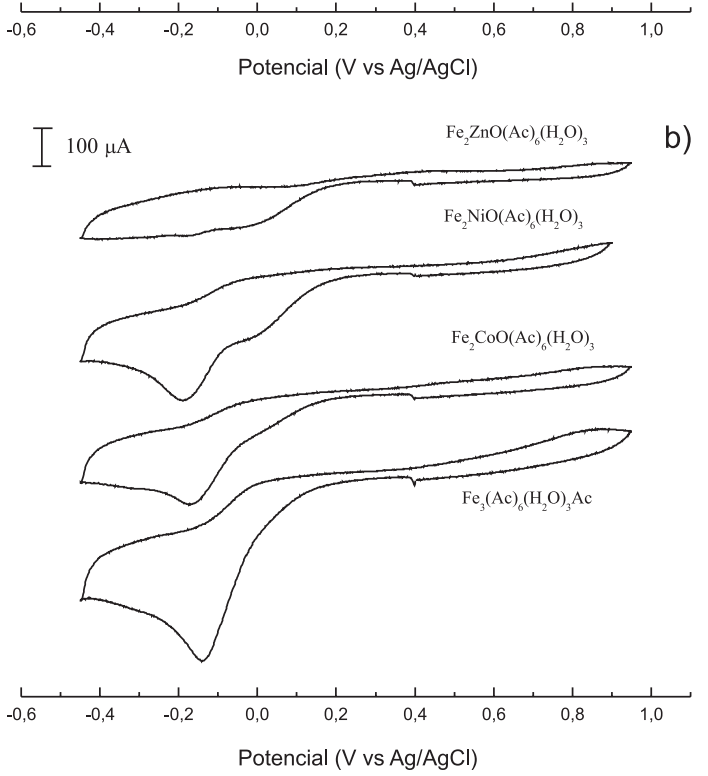

Figura 3. Voltamogramas cíclicos (A) obtidos para os precursores usados nas sínteses e (B) trinucleares homo e heteronucleares (concentrações iguais). Velocidade de varredura igual a $100 \mathrm{mV} / \mathrm{s}$

dos metais já se encontra reduzido.

A questão da decomposição do trinuclear com a redução foi discutida com os alunos, enfatizando-se as diferenças nas cinéticas de troca de ligantes. Antes da discussão, foi apresentado aos alunos um artigo que foi escolhido por apresentar um voltamograma usando o mesmo eletrodo de referência, além da atribuição dos processos de um complexo trinuclear de rutênio. ${ }^{25}$ Facilmente o aluno consegue perceber a diferença entre os trinucleares, pois o composto de rutênio pode ser reduzido por dois processos monoeletrônicos reversíveis e também pode ser oxidado reversivelmente, sem ocorrência perceptível de reações acopladas, dado o fato de ele ser mais inerte que seus análogos contendo ferro.

Um trabalho que pode ser usado como referência pelos alunos é o artigo de Mabbott, ${ }^{26}$ segundo o qual a reversibilidade do processo 
eletroquímico de oxidação e redução fundamenta-se na cinética de reações químicas acopladas à oxidação e/ou à redução. Tomemos uma reação genérica em que a espécie $\mathrm{O}$ se reduz para $\mathrm{R}$ que, em seguida, é consumido para formar $\mathrm{Z}$ numa reação química acoplada.

$$
\begin{gathered}
\mathrm{O}+\mathrm{n} e \rightarrow \mathrm{R} \text { (reação } 1) \\
\mathrm{R} \rightarrow \mathrm{Z} \text { (reação } 2)
\end{gathered}
$$

Dessa forma, se a reação 2 for rápida e a velocidade de varredura (v) for lenta, parte das espécies $\mathrm{R}$ é transformada em $\mathrm{Z}$ e menos $\mathrm{R}$ estará disponível para se oxidar de volta a $\mathrm{O}$, causando diminuição da corrente anódica. Se a reação 2 acontecer numa velocidade muito maior que $v$, todo $\mathrm{R}$ será convertido e a onda anódica poderá simplesmente não aparecer.

A hipótese da estabilidade dos trinucleares estar associada às labilidades dos íons foi discutida com os alunos utilizando as constantes de troca da água nos aquocomplexos com fórmula $\left[\mathrm{M}\left(\mathrm{H}_{2} \mathrm{O}\right)_{6}\right]^{\text {n+ }}$ : $\mathrm{Fe}(\mathrm{II})^{27} \log k=6,64 ; \mathrm{Fe}(\mathrm{III})^{26} \log k=2,20 ; \mathrm{Ru}(\mathrm{II})^{28} \log k=-1,84$; $\mathrm{Ru}(\mathrm{III})^{29,30} \log k=-5,46$. A comparação das constantes de velocidade mostra claramente a inércia dos complexos de rutênio $\left(k<1 \mathrm{~s}^{-1}\right)$ e o aumento da constante de troca (associada à labilidade) de cerca de 10.000 vezes quando os íons Ru(III) são reduzidos. ${ }^{31}$ Tendo feito isso, um segundo artigo $^{32}$ foi apresentado mostrando que ao se utilizar um ligante polidentado para a obtenção da estrutura $\mathrm{Fe}_{3} \mathrm{O}^{+}$, duas reduções monoeletrônicas reversíveis são observadas, confirmando a hipótese anterior. Esse segundo artigo também oferece ao professor a opção de abordar o efeito quelato com os alunos.

Aspectos incomuns ligados à caracterização eletroquímica também podem ser estudados pelos alunos usando esses trinucleares. Variando-se a velocidade de varredura, os alunos puderam obter uma correlação linear entre a corrente de pico catódica e a raiz quadrada da velocidade de varredura, a mesma situação para o caso da redução reversível obtida com soluções de hexacianoferrato(III) de potássio. ${ }^{32}$ Normalmente essa relação é considerada como uma prova da reversibilidade de um processo eletroquímico, porém também é válida para processos irreversíveis. ${ }^{33}$

\section{CONCLUSÕES}

Um procedimento de síntese de complexos trinucleares homo e heterometálicos de ferro e outros metais de transição foi apresentado, de modo que essas sínteses possam ser realizadas por alunos de graduação usando reagentes usuais e de baixo custo. A caracterização desses materiais usando a técnica de infravermelho em pastilhas de $\mathrm{KBr}$ oferece um bom exemplo de como o professor pode discutir os modos de coordenação de ligantes em ponte e, também, os modos normais de vibração envolvendo íons de metais de transição. Os experimentos propostos puderam ser usados para discutir diversos assuntos ligados à química de coordenação como, por exemplo, estrutura e simetria, reversibilidade de reações de oxirredução, labilidade/ inércia, efeito trans, efeito quelato, diferenças entre as propriedades de metais da primeira e da segunda séries de transição, além de aspectos ligados à caracterização eletroquímica de compostos de coordenação. A avaliação dos alunos a respeito dos procedimentos experimentais e conceitos utilizados foi positiva e, quando questionados sobre o sucesso da aula prática, de modo geral, os alunos responderam que a aula experimental foi importante para a consolidação de conceitos importantes da ementa da disciplina.

\section{AGRADECIMENTOS}

Ao Prof. C. U. Davanzo (IQ-UNICAMP) pelas valiosas discus- sões e sugestões. S. A. V. Jannuzzi agradece à FAPESP e R. S. Santos à CAPES pelas bolsas concedidas. A. L. B. Formiga agradece ao CNPq pelo financiamento (proc. no. 307881/2007-5 e 479415/2009-9).

\section{REFERÊNCIAS}

1. Nakamoto, T.; Katada, M.; Sano, H.; Inorg. Chim. Acta 1999, 291, 127; Powell, G.; Richens, D. T.; Powell, A. K.; Inorg. Chim. Acta 1993, 213, 147; Jr., C. E. S.; Steinmetz, G. R.; J. Am. Chem. Soc. 1985, 107, 6124; Cotton, F. A.; Lewis, G. E.; Mott, G. N.; Inorg. Chem. 1982, 21, 3316; Müller, A.; Jostes, R.; Cotton, F. A.; Angew. Chem., Int. Ed. 1980, 19, 875.

2. Cotton, F.A.: Wilkinson, G.; Advanced Inorganic Chemistry, $5^{\text {th }}$ ed., Wiley: New York, 1988.

3. Greenwood, N. N.: Earnshaw, A.; Chemistry of the Elements, $2^{\text {nd }}$ ed., Butterworth-Heineman: Oxford, 1997.

4. Orgel, L. E.; Nature 1960, 187, 504.

5. Cotton, F. A.; Norman Jr., J. G.; Inorg. Chim. Acta 1972, 6, 411.

6. Aleixou, A. D. P.; Dovidauskas, S.; Toma, H. E.; Quim. Nova 2000, 23, 785 .

7. Dovidauskas, S.; Toma, H. E.; Araki, K.; Sacco, H. C.; Iamamoto, Y.; Inorg. Chim. Acta 2000, 305, 206.

8. Winnischofer, H.; Otake, V. Y.; Dovidauskas, S.; Nakamura, M.; Toma, H. E.; Araki, K.; Electrochim. Acta 2004, 49, 3711.

9. Formiga, A. L. B.; Nogueira, A. F.; Araki, K.; Toma, H. E; New J. Chem. 2008, 32, 1167.

10. Henschel, A.; Gedrich, K.; Kraehnert, R.; Kaskel, S.; Chem. Commun. 2008, 35, 4192.

11. Chowdhury, P.; Bikkina, C.; Gumma, S. J.; Phys. Chem. C 2009, 113, 6616; Hamon, L.; Serre, C.; Devic, T.; Loiseau, T.; Millange, F.; Ferey, G.; De Weireld, G.; J. Am. Chem. Soc. 2009, 131, 8775.

12. Rajaraman, G.; Murugesu, M.; Sanudo, E.; Soler, M.; Wernsdorfer, W.; Helliwell, M.; Muryn, C.; Raftery, J.; Teat, S.; Christou, G.; Brechin, E. ; J. Am. Chem. Soc. 2004, 126, 15445; Moushi, E. E.; Stamatatos, T. C.; Wernsdorfer, W.; Nastopoulos, V.; Christou, G.; Tasiopoulos, A. J.; Angew. Chem., Int. Ed. 2006, 45, 7722; Viciano-Chumillas, M.; Tanase, S.; Mutikainen, I.; Turpeinen, U.; de Jongh, L. J.; Reedijk J.; Inorg. Chem. 2008, 47, 5919.

13. Vrubel, H.; Hasegawa, T.; de Oliveira, E.; Nunes, F. S.; Inorg. Chem. Commun. 2006, 9, 208.

14. Weinland, R.; Holtmeier, H.; Z. Anorg. Chem. 1928, 173, 49.

15. Blake, A. B.; Yavari, A.; Hatfield, W.; Sethulekshmi, C. N.; J. Chem. Soc. Dalton Trans. 1985, 2509.

16. Miccichè, F.; Long, G. J.; Shahin, A. M.; Grandjean, F.; Ming, W.; van Haveren, J.; van der Linde, R.; Inorg. Chim. Acta 2007, 360, 535.

17. Sanakis, Y.; Boudalis, A. K.; Tuchagues, J.-P.; C. R. Chim. 2007, 10, 116.

18. Cabe lembrar que assumimos uma simetria local $\mathrm{D}_{3 \mathrm{~h}}$ para a unidade $\mathrm{M}_{3} \mathrm{O}$, quando os três íons metálicos são iguais.

19. O professor pode explorar a existência de um análogo orgânico contendo um oxigênio planar $\left(\mathrm{R}=\mathrm{OR}^{+}\right)$e aromático. De fato, os heterocíclicos derivados do íon pirílio de origem natural (análogo oxigenado da piridina) são pigmentos largamente encontrados em flores.

20. Clayden, J.; Greeves, N.; Warren, S.; Wothers, P.; Organic Chemistry, Oxford University Press: Oxford, 2001.

21. Nakata, K.; Nagasawa, A.; Soyoma, N.; Sasaki, Y.; Ito, T.; Inorg. Chem. 1991, 30, 1575.

22. Santos, J. M. T.; Silva, R. R.; Formiga, A. L. B.; Tinoco, L. W.; Figueroa-Villar, J. D.; Chem. Phys. 2004, 306, 143.

23. Johnson, M. K.; Powell, D. B.; Cannon, R. D.; Spectrochim. Acta, Part A 1981, 37, 995.

24. White, R. P.; Wilson, L. M.; Williamson, D. J.; Moore, G. R.; Jayasooriya, U. A.; Cannon, R. D.; Spectrochim. Acta, Part A 1990, 46, 917. 
25. Abe, M.; Sato, A.; Inomata, T.; Kondo, T.; Uosaki, K.; Sasaki, Y.; Dalton Trans. 2000, 2693.

26. Mabbott, G. A.; J. Chem. Educ. 1983, 60, 697.

27. Tobe, M. L. Em Comprehensive Coordination Chemistry; Wilkinson, G.; Gillard, R. D., McCleverty, J. A., eds.; Oxford:Pergamon, 1987, vol. 1, cap. 7.

28. Abram, U. Em ref. 27, vol. 4, cap. 45.

29. Rapaport, I.; Helm, L.; Merbach, A. E.; Bernhard, P.; Ludi, A.; Inorg. Chem. 1988, 27, 873.
30. Powell, G.; Richens, D. T.; Inorg. Chim. Acta 1993, 213, 147.

31. O professor ainda pode explorar neste ponto o efeito trans da ponte $\mu_{3}$-oxo. A troca do ligante aquo no trinuclear $\left[\mathrm{Ru}_{3} \mathrm{O}\left(\mathrm{CH}_{3} \mathrm{CO}_{2}\right)_{6}\left(\mathrm{H}_{2} \mathrm{O}\right)_{3}\right]^{+}$ é cerca de 3 ordens de grandeza mais rápida quando comparada ao aquocomplexo (vide ref. 29).

32. Saalfrank, R. W.; Trummer, S.; Krautscheid, H.; Schünemann, V.; Trautwein, A. X.; Hien, S.; Stadler, C.; Daub, J.; Angew. Chem., Int. Ed. 1996, 35, 2206

33. Silva, R. C.; Souza, I. G.; Quim. Nova 1998, 21, 477. 